\title{
Analysis of Distribution Generation Influences on the Vol- tage Limit Violation Probability of Distribution Line
}

\author{
Lu Zhang, Wei Tang, Muke Bai, Pengwei Cong \\ College of Information and Electrical Engineering, China Agriculture University, Beijing, China \\ Email:602996165@qq.com
}

Received April, 2013

\begin{abstract}
Considering the time-sequence characteristic and randomness of load and natural resources, this paper studies on the distributed generation (DG) impacts on voltage limit violation probability of distribution lines. The time-sequence characteristic and randomness of load, wind and photovoltaic (PV) generation are analyzed; the indices and risk levels of voltage limit violation probability of node and distribution line are proposed. By using probabilistic load flow based on semi-invariant method, the impact degrees of voltage limit violation are calculated with different distributed power penetration levels, different seasons, different time periods, different allocation ratio between the wind power and PV power. Voltage limit violation laws of distribution line, which are concluded by IEEE33 bus system simulation, are very helpful to guide the voltage regulation of distribution line including distributed generation.
\end{abstract}

Keywords: Distribution Generation; Distribution Line; Voltage Limit Violation Probability; Time-sequence Characteristic; Randomness

\section{Introduction}

Renewable energy is paid more attention and application for its cleanness and environment protection in recent years, so the research of distributed generation (DG) has become focus. Effect of DG on voltage of distribution line becomes serious with the increasing DG penetration levels. As a result, the analysis of voltage limits violation, which caused by DG, has important significance for ensuring economic and safe operation of power grid.

There have been some researches on impact of voltage limit violation of DG connected to power grid. Effect of DG connected to transmission grid is analyzed by sensitivity analysis method [1]. A probabilistic assessment method was proposed [2], which adapt to different wind speed levels in transmission grid. Reference [3] analyzed impact of DG on voltage profiles using triangular load model. Impact of photovoltaic (PV) power on voltage was analyzed [4, 5]. Summarizing the literature [3-5], only maximum output of DG and peak load was considered and the indices were calculated by certain method. But in fact, load and output of DG fluctuate with seasons significantly; daily load has large difference between peak and valley, which also has strong uncertainty. Therefore, the previous works are considered inadequately.

This paper comprehensively considers randomness and time-sequence characteristic of load and DG. The organization is as follows: Time-sequence characteristic and randomness of load and DG is introduced in Section 2. Indices of distribution line voltage limit violation probability and calculation method are proposed in Section 3. Analysis of distribution line voltage limit violation is presented in Section 4, followed by conclusions in Section 5 .

\section{Time-Sequence Characteristic and Randomness of Load and DG}

\subsection{Time-Sequence Characteristic and Randomness of Load}

Load fluctuates in timing. The variation of load is different in four seasons a year. The differences of load are great in different periods a day in same season, but there are significant regularities. The period of peak happens nearly at the same time every day and so does the period of valley. Time-sequence characteristic of load in typical day of different seasons is shown in Figure 1.

On the other hand, load has randomness because of the error of load forecasting and the causal factors, which cause the load curve is not exactly the same as typical day.

Load curves could be divided according to change law of load time-sequence characteristics a day, and 24 hours a day are segmented as shown in Table 1. Normal distribution mean value and standard deviation could be obtained by statistical calculations of historical load data. 


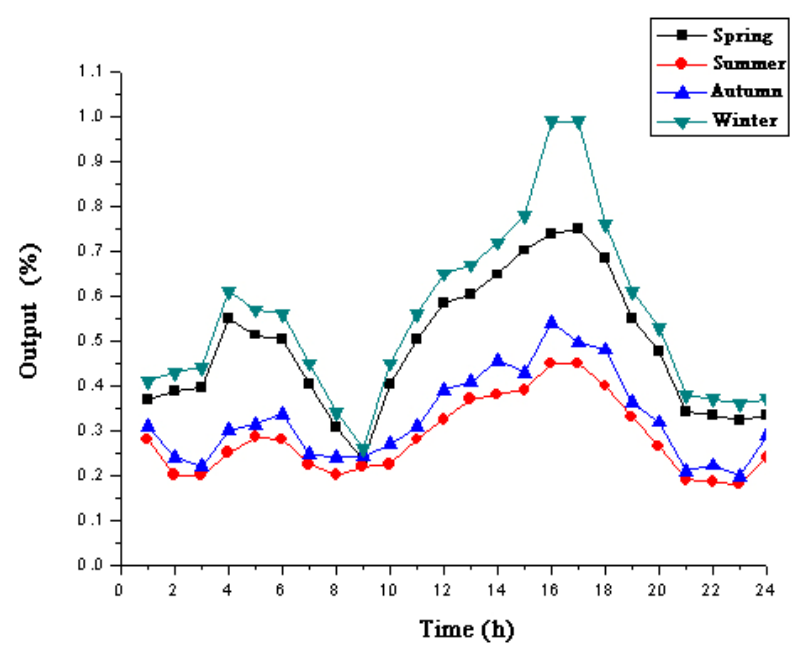

Figure 1. Time-sequence characteristic of load.

Table 1. Load time-interval.

\begin{tabular}{ccccc}
\hline periods & Early in morning & Morning & Noon & Afternoon \\
\hline Time & $(6-8]$ & $(8-11]$ & $(11-14]$ & $(14-17]$ \\
Periods & Evening & Night & Late at night & \\
Time & $(17-19]$ & $(19-23]$ & $(23-6]$ & \\
\hline
\end{tabular}

\subsection{Time-Sequence Characteristic and Randomness of Wind Generation}

Wind turbine generator (WTG) power, which changes with wind speed, has apparent randomness. Affected by natural conditions, wind speed has a very strong uncertainty. Waybill distribution is generally used as the statistical description of the wind speed. The relationship of WTG power and wind speed can be shown as a curve of output characteristic.

WTG power also has time-sequence characteristic. There is a big difference of wind speed between different seasons [6]. In different periods a day, speed changes also have a statistical characteristic. Wind speed timing characteristic curve of different seasons could be obtained by meteorological data, as shown in Figure 2.

\subsection{Time-Sequence Characteristic and Randomness of PV Generation}

PV generator output has a direct relationship with the solar intensity. Sunlight radiation intensity depends on solar altitude and attenuation by clouds. The solar altitude changing with time can be expressed by a certain function. So PV generator output has time-sequence characteristic [7], as shown in Figure 3, which has little difference between different seasons. On the other hand, $\mathrm{PV}$ generator output is random because of the random attenuation by clouds.

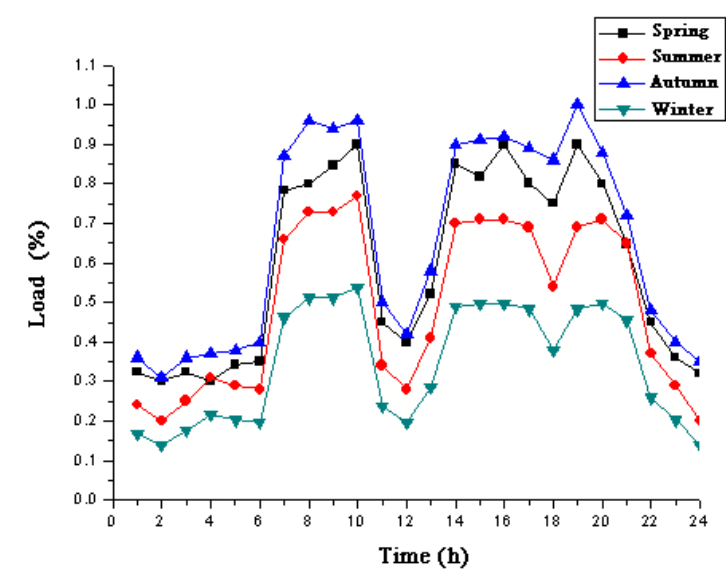

Figure 2. Time-sequence characteristic of WTG power.

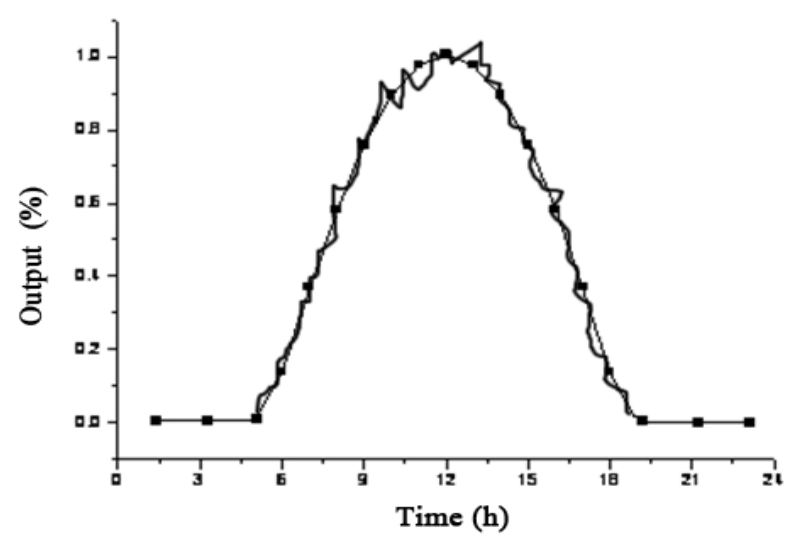

Figure 3. Time-sequence characteristic of PV.

It can be concluded from above that:

1) All of load, WTG and PV power have time-sequence characteristic and randomness.

2) The moment of maximum load is not the same with maximum WTG and PV power output, and the indices calculated based on a certain value or certain levels of load and DG output have large differences with that in actual situation, so the results are not accurate.

3) Both load and DG output have randomness, certain indices can't fully reflect the potential risk in power grid.

Therefore, it is important to calculate the voltage limit violation probability in different periods of the day comprehensively considering time-sequence characteristic and randomness of the load and DG in order to really reflect the situation of voltage in power grid.

\section{Indices of Distribution Line Voltage Limit Violation Probability}

\subsection{Voltage Limit Violation Probability Indices of Node and Distribution Line}

According to China national standards, the allowable deviation of node voltage in $10 \mathrm{kV}$ distribution lines is 
the $\pm 7 \%$ of rated voltage, so when the node voltage is higher than $1.07 \mathrm{pu}$ or lower than $0.93 \mathrm{pu}$, voltage violates limits. Voltage upper or lower limit violation probability means the possibility of node voltage higher than $1.07 \mathrm{pu}$ or below $0.93 \mathrm{pu}$.

Distribution line voltage limit violation probability is integrated statistics of node voltage. Assume that $N$ nodes voltage limit violation probability is $P_{k}^{t}(k=$ $1,2 \ldots N)$, where $t$ is period. The distribution line voltage limit violation probability in a period can be expressed as:

$$
P^{t}=1-\prod_{k=1}^{N}\left(1-P_{k}^{t}\right)
$$

Distribution line voltage upper limit violation probability is calculated by:

$$
P_{u p}^{t}=1-\prod_{i=1}^{N}\left(1-P_{i u p}^{t}\right)
$$

where $P_{i u p}^{t}$ is the $i$ th node in the distribution line voltage upper limit violation probability in $t$ period. Line voltage lower limit violation probability is calculated by:

$$
P_{\text {down }}^{t}=1-\prod_{i=1}^{N}\left(1-P_{i d o w n}^{t}\right)
$$

where $P_{\text {idown }}^{t}$ is the $i$ th node in the distribution line voltage lower limit violation probability in $t$ period.

If voltage of any node is over limit, the distribution line is in risk state. According to the severity level, the state is divided into no risk, mild risk, medium risk and risk of severe, as shown in Table 2.

\subsection{Calculation Method of Distribution Line Voltage Limit Violation Probability Based on Probabilistic Power Flow}

Considering the randomness of the load and DG, this paper uses probabilistic power flow to obtain probability value of voltage limit violation. Probabilistic power flow combined semi-invariant and Gram-Charlier expanding is used in order to avoid the large amount calculation of Monte Carlo method. The algorithm laniaries equations of bus injected power and line flow by Taylor series expanding at normal operation point:

$$
\left\{\begin{array}{l}
S_{0}+\Delta S=f\left(X_{0}+\Delta X\right)=f\left(X_{0}\right)+J_{0} \Delta X+\ldots . \\
Z_{0}+\Delta Z=g\left(X_{0}+\Delta X\right)=g\left(X_{0}\right)+G_{0} \Delta X+\ldots
\end{array}\right.
$$

where $S_{0}, X_{0}$ and $Z_{0}$ are expectation of bus injection power, state variable and line flow, respectively; $\Delta S, \Delta X$ and $\Delta Z$ are their random disturbance.

Assuming that loads of nodes random change is mutually independent, winds speed and sunlight intensity are mutually independent, wind speed obeys distribution of Waybill and sunlight intensity obeys beta distribution in each period. Algebraic operations of semi-invariant take the place of convolution calculation, and then all older semi-invariant of unknown quantity are obtained. Finally the probability density function of state variables is calculated by Gram-Charlier series expansion method.

Detailed procedures are as follows:

1) Enter the basic data of the power grid, including grid structure and the basic parameters of the load and DG.

2) Obtain the load and power curve of typical day in different seasons by meteorological data or historical data; calculate the time-sequence characteristic parameters of load and DG in different periods and different seasons.

3) Calculate nodes voltage limit violation probability in different periods and different seasons by using probabilistic flow based on semi-invariant.

4) Calculate voltage limit violation probability of distribution line by nodes' in different periods and seasons.

5) Evaluate the risk level.

\section{Analysis of Distribution Line Voltage Limit Violation Probability}

IEEE33 bus distribution system is used as example in this paper. In 14, 23, 29 nodes join WTG and PV, as shown in Figure 4, and related data are shown in Table 3, Table 4.

The penetration level can be changed by accessing different numbers of DG. Load obeys the normal distribution with $3 \%$ variance, the bus 0 voltage taken $1.02 \mathrm{pu}$, and the line maximum load is $7430 \mathrm{~kW}$.

\subsection{Effect of Load Rate and DG Penetration}

According to load rate of $20 \%, 50 \%, 80 \%$, this paper divides distribution line into three types: light load, normal load and high load. Voltage upper and lower limit violation probability of distribution lines is calculated in different penetration levels, and the results are shown in Figure 5 - Figure 7, Table 5 - Table 7.

Table 5 and Figure 5 show that: the voltage of light load line without DG isn't over limit. After joining DG, with the increase of the penetration, voltage begins to exceed upper limit, which mainly appears near DG and

Table 2. Risk levels of voltage limit violation probability.

\begin{tabular}{cc}
\hline limit violation probability(\%) & Risk level \\
$(0-0.1]$ & No risk \\
$(0.1-20]$ & Mild risk \\
$(20-50]$ & Medium risk \\
More than 50 & Risk of severe \\
\hline
\end{tabular}


Table 3. Characteristics of wind farms.

\begin{tabular}{ccccccc}
\hline $\begin{array}{c}\text { Rated } \\
\text { capacity/MW }\end{array}$ & $\begin{array}{c}\text { Cut-in } \\
\text { speed/(m/s) }\end{array}$ & $\begin{array}{c}\text { Rated } \\
\text { speed/(m/s) }\end{array}$ & $\begin{array}{c}\text { Cut-out } \\
\text { speed/(m/s) }\end{array}$ & $k$ & $c$ \\
\hline 0.4 & 3 & 14 & 25 & 1.741 & 5.612 \\
\hline
\end{tabular}

Table 4. Characteristics of solar parks.

\begin{tabular}{ccccc}
\hline $\begin{array}{c}\text { Square } \\
\text { total area/m } / \mathrm{m}^{2}\end{array}$ & $\begin{array}{c}\text { Photoelectrical } \\
\text { conversion efficiency/\% }\end{array}$ & $\begin{array}{c}\text { Highest } \\
\left.\text { irradiance/(W/m }{ }^{2}\right)\end{array}$ & $\alpha$ & $\beta$ \\
\hline 800 & 14 & 600 & 0.85 & 0.85 \\
\hline
\end{tabular}

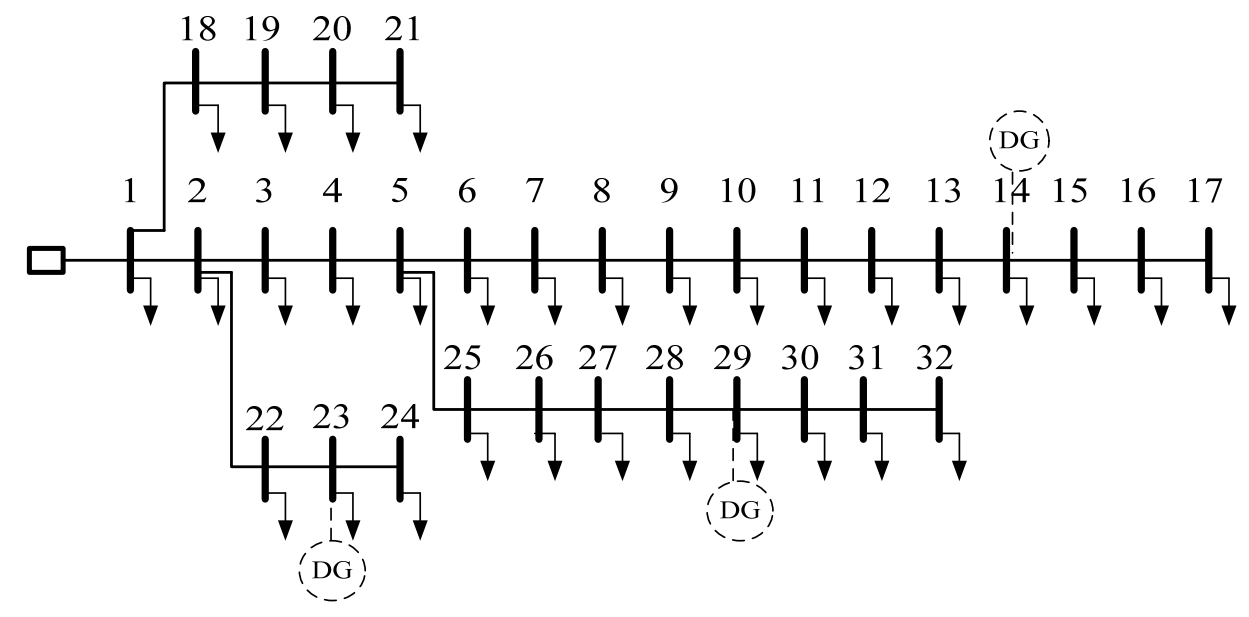

Figure 4. IEEE33 bus system including DG.

the small load nodes. If DG is installed near bus 0 , nodes voltage in front may be over upper limit. When penetration is more than $30 \%$, the probability of voltage upper limit violation is higher than $20 \%$, which is medium risk. When penetration level increases to $40 \%$, the probability of voltage upper limit violation reaches $55 \%$, which is risk of severe.

Table 5. Voltage limit violation probability of light load line.

\begin{tabular}{cccc}
\hline & \multicolumn{3}{c}{ Light load line $(1634 \mathrm{~kW})$} \\
\cline { 2 - 4 } $\begin{array}{c}\text { Penetration rate } \\
\text { of DG(\%) }\end{array}$ & $\begin{array}{c}\text { Probability of } \\
\text { lower limit(\%) }\end{array}$ & $\begin{array}{c}\text { Probability of } \\
\text { upper limit(\%) }\end{array}$ & $\begin{array}{c}\text { Total over-limit } \\
\text { probability(\%) }\end{array}$ \\
\hline 10 & 0 & 0 & 0 \\
15 & 0 & 0 & 0 \\
20 & 0 & 0.16 & 0.16 \\
25 & 0 & 0.52 & 0.52 \\
30 & 0 & 9.23 & 9.23 \\
35 & 0 & 34.4 & 23.41 \\
40 & 0 & 55.0 & 55.01 \\
\hline
\end{tabular}

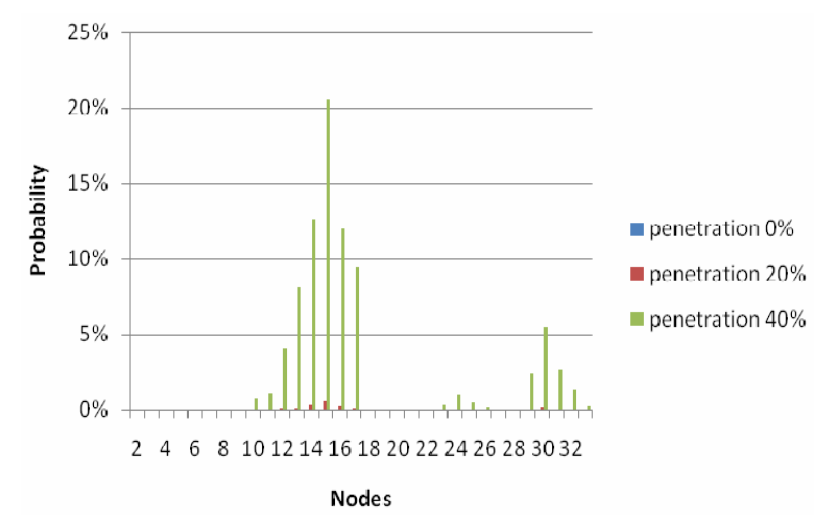

Figure 5. Voltage limit violation probability of light load line.

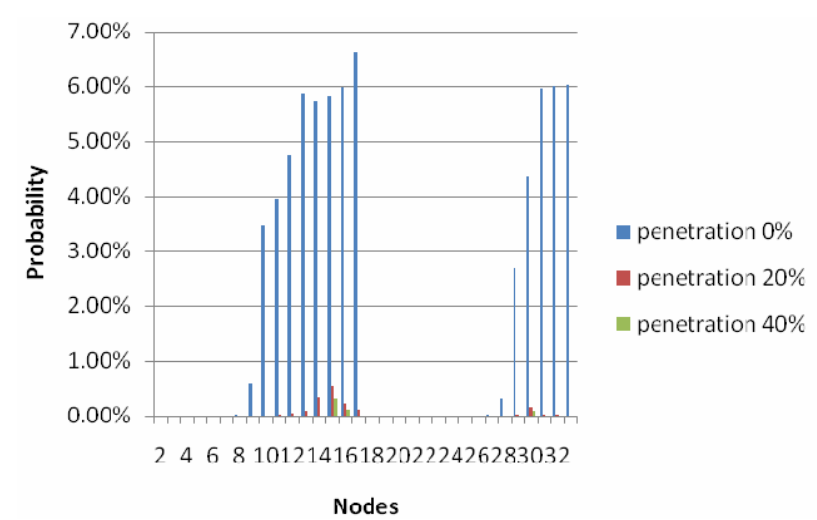

Figure 6. Voltage limit violation probability of normal load line. 
Table 6. Voltage limit violation probability of normal load line.

\begin{tabular}{cccc}
\hline & \multicolumn{3}{c}{ Normal load line (4086kW) } \\
\cline { 2 - 4 } $\begin{array}{c}\text { Penetration } \\
\text { of DG(\%) }\end{array}$ & $\begin{array}{c}\text { Probability of } \\
\text { lower limit(\%) }\end{array}$ & $\begin{array}{c}\text { Probability of } \\
\text { upper limit(\%) }\end{array}$ & $\begin{array}{c}\text { Total over-limit } \\
\text { probability(\%) }\end{array}$ \\
\hline 0 & 41.85 & 0 & 41.85 \\
10 & 12.10 & 0 & 12.10 \\
15 & 3.12 & 0 & 3.12 \\
20 & 1.70 & 0 & 1.73 \\
25 & 0.95 & 0.07 & 0.07 \\
30 & 0 & 0.11 & 0.09 \\
35 & 0 & 0.34 & 0.34 \\
40 & 0 & 0.85 & 0.85 \\
\hline
\end{tabular}

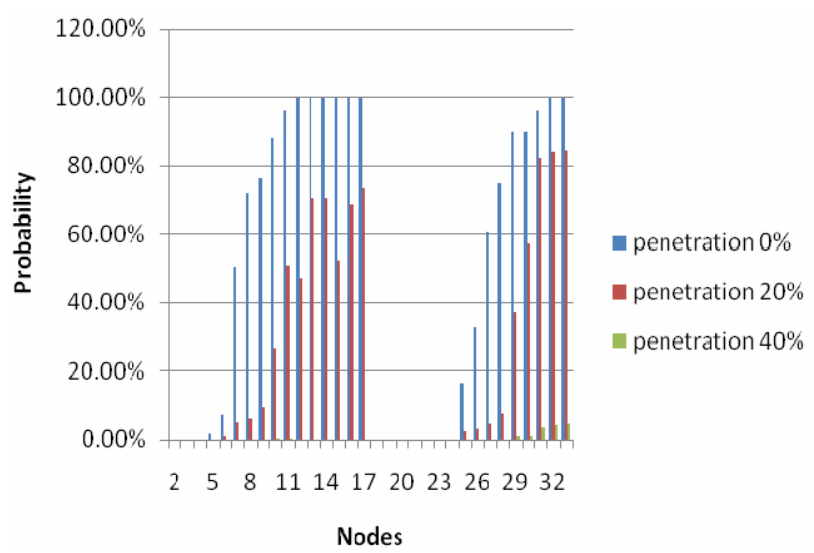

Figure 7. Voltage limit violation probability of high load line.

Table 7. Voltage limit violation probability of high load line.

\begin{tabular}{cccc}
\hline & \multicolumn{3}{c}{ High load line (6538kW) } \\
\cline { 2 - 4 } Penetration \\
\cline { 2 - 4 } & 100 & 0 & 100 \\
& $\begin{array}{c}\text { Probability of } \\
\text { lower limit(\%) }\end{array}$ & $\begin{array}{c}\text { Probability } \\
\text { of upper limit(\%) }\end{array}$ & $\begin{array}{c}\text { Total over-limit } \\
\text { probability(\%) }\end{array}$ \\
\hline 0 & 100 & 0 & 100 \\
10 & 99.99 & 0 & 99.99 \\
15 & 93.95 & 0 & 93.95 \\
20 & 84.50 & 0 & 84.50 \\
25 & 58.40 & 0.006 & 58.41 \\
30 & 24.33 & 0.01 & 24.34 \\
35 & 13.17 & 0.02 & 13.19 \\
40 & & &
\end{tabular}

Table 6 and Figure 6 show that: for normal load lines, the probability of nodes, which located in the end of the line or large load, voltage lower limit violation is about $6 \%$ without DG, and the whole line probability of over limit is about $41.85 \%$, which is medium risk. With the increasing penetration levels, the probability of lower limit violation is reduced gradually. When the penetration is $30 \%$, probability of lower limit violation is $0 \%$, while the whole line probability of upper limit violation is $0.11 \%$. If continue increasing the penetration of DG, the upper limit violation probability continues to increase.

Table 7 and Figure 7 show that: for high load lines, nodes located in the end of the line voltage lower limit violation probability nearly $100 \%$. When penetration increases to $30 \%$, the probability of node voltage over lower limit significantly alleviates, and the end node voltage lower limit violation probability is no more than $5.92 \%$.

\subsection{Effect of Seasons and Periods}

DG output varies with season and period. WTG output in winter is larger than other seasons, and its maximum periods of a day are afternoon and late at night. The output of PV has time-sequence characteristic, and it doesn't change a lot in different seasons. The periods of maximum output of PV are noon and afternoon, and PV output is nearly zero after evening. Distribution line in example is served for agriculture load, which is busy in autumn and free in winter, so load is high in autumn and light in winter. Load and output of DG are calculated according to the method given above; penetration rate of DG is $30 \%$. Because of page limit, this paper only give the calculation result of autumn shown in Table 8 and Figure 8, winter in Figure 9.

Table 8. Voltage limit violation probability of time-sequence characteristic in autumn.

\begin{tabular}{|c|c|c|}
\hline Periods & Indices & $\begin{array}{c}\text { Calculated } \\
\text { values }\end{array}$ \\
\hline \multirow{3}{*}{$\begin{array}{l}\text { Early in } \\
\text { morning }\end{array}$} & Probability of upper limit（\% ) & 0.006 \\
\hline & Probability of lower limit（\%) & 35.860 \\
\hline & Total over-limit probability（\%) & 35.866 \\
\hline \multirow{3}{*}{ Morning } & Probability of upper limit (\%) & 0.004 \\
\hline & Probability of lower limit（\% ) & 94.685 \\
\hline & Total over-limit probability（\%) & 94.689 \\
\hline \multirow{3}{*}{ Noon } & Probability of upper limit（\%) & 1.434 \\
\hline & Probability of lower limit（\% ) & 0.001 \\
\hline & Total over-limit probability（\%) & 1.434 \\
\hline \multirow{3}{*}{ Afternoon } & Probability of upper limit (\%) & 0.004 \\
\hline & Probability of lower limit（\%) & 70.160 \\
\hline & Total over-limit probability（\%) & 70.164 \\
\hline \multirow{3}{*}{ Evening } & Probability of upper limit（\%) & 0.004 \\
\hline & Probability of lower limit（\% ) & 96.045 \\
\hline & Total over-limit probability（\%) & 96.049 \\
\hline \multirow{3}{*}{ Night } & Probability of upper limit（\% ) & 0.005 \\
\hline & Probability of lower limit (\%) & 93.675 \\
\hline & Total over-limit probability（\%) & 93.680 \\
\hline \multirow{3}{*}{$\begin{array}{l}\text { Late at } \\
\text { night }\end{array}$} & Probability of upper limit（\% ) & 0.017 \\
\hline & Probability of lower limit（\%) & 0.002 \\
\hline & Total over-limit probability（\%) & 0.019 \\
\hline
\end{tabular}




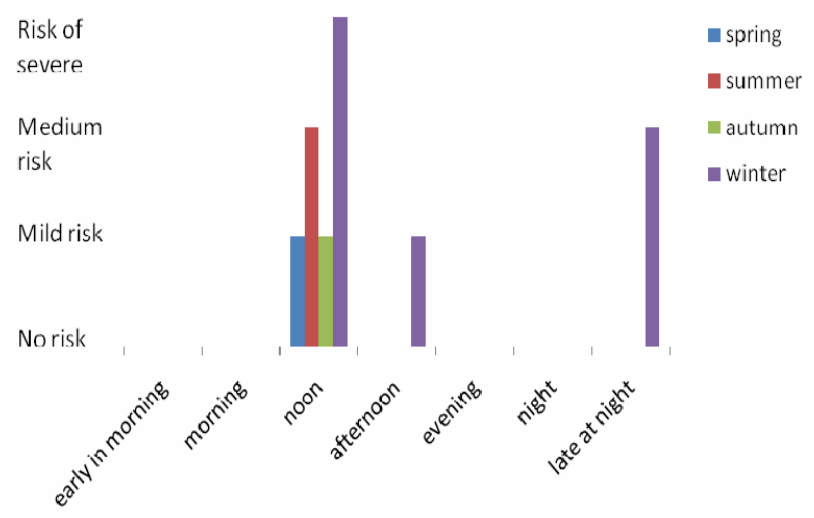

Figure 8. Risk levels of voltage over upper limit in different seasons and time periods.

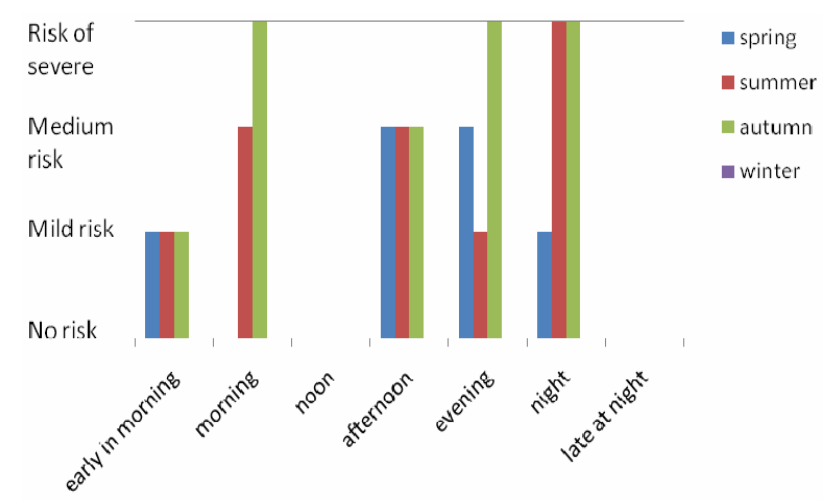

Figure 9. Risk levels of voltage over lower limit in different seasons and time periods.

Table 8 shows that: in autumn, load is large but DG output is low, so the voltage lower limit violation probability is generally higher than other seasons. In winter, load is low but DG output is large, so the voltage upper limit violation probability is generally higher than other seasons. Output of PV is largest at noon during a day, but load is small in lunch break, so the voltage of line is easily over upper limit. At noon in winter, the voltage upper limit violation probability of the line is $53.275 \%$, which is risk of severe. In the Afternoon in winter, both PV and WTG output are large, as the load in winter is generally low, so the probability of upper limit violation is $10.14 \%$, which is mild risk. Load is generally small late at night, but output of WTG is large, so voltage upper limit violation probability is $11.015 \%$, which is mild risk. Load in periods of morning, afternoon, evening and night is large, so probability of over lower limit is high if DG output is small.

Figure 8 shows that: noon is high-frequency period of over upper limit, especially in winter and summer. Figure 9 shows that: morning, evening and night are highfrequency periods of over lower limit, especially in autumn and summer. Although load in afternoon is large, DG output is larger, so the level of risk is reduced.

\subsection{Effect of Allocation Ratio between The Wind Power and PV Power}

Because of the different natural resources in different area, allocation ratio between the wind power and PV power is different. Affected by local nature resources, allocation ratio between the wind power and PV power will change the probability of voltage limit violation if penetration is fixed. Probabilities of voltage limit violation in different allocation ratios between the wind power and PV power results are shown in Table $\mathbf{9}$.

Table 9 shows that: the allocation ratio affects the voltage limit violation probability and the degree of effect depends on local natural resources. In the area major in WTG, because of the small load and the large output of WTG late at night, the probability of voltage upper limit violation is $38.66 \%$, which is medium risk. In the area major in PV, probability of voltage upper limit violation at noon is $70.99 \%$. But PV output is almost 0 from evening to late at night, from 17 to 19 o'clock, the probability of line voltage over lower limit is $40.67 \%$, and from 19 to 23 o'clock, the probability is $51.34 \%$. In area where allocation ratio is balanced, risk of severe period won't appear during the whole day.

\section{Conclusions}

This paper comprehensively considers time-sequence characteristic and randomness of load and DG, and researches the effect of DG on distribution line voltage limit violation probability with different penetration levels, different seasons, different periods, different allocation ratio of wind and light. The result is as follows:

1) For different load ratio distribution line, DG penetration has large affect on probability of voltage limit violation. For light load lines, adding DG improves the overall distribution line voltage levels, and the risk level increases with the increasing penetration rate. For high load lines, nodes in the end of the line voltage over lower limited probability is high, and with the increasing penetration levels, the probability will be reduced, and the risk level dropped. Penetration rate of $30 \%$ can effectively alleviate the voltage-limit.

2) For different seasons and periods, probability of nodes' voltage limit violation is different. Transformer tap of substation and voltage regulator in distribution line should be adjusted based on the time-sequence characteristic of load and DG according to different seasons and periods.

3) For different areas, the natural resources are different greatly, so it makes a great difference in voltage limit violation probability. Proper allocation ratio between the wind power and PV power could effectively alleviate the risk levels. 
Table 9. Voltage limit violation probability of time-sequence characteristics in different allocation ratios between the wind power and PV power.

\begin{tabular}{|c|c|c|c|c|c|c|}
\hline Periods & Indices & $\begin{array}{l}\text { ration between wind } \\
\text { power and PV pow- } \\
\text { er(\%) } 0 / 100\end{array}$ & $\begin{array}{l}\text { ation between wind } \\
\text { power and PV } \\
\text { power(\%) 30/70 }\end{array}$ & $\begin{array}{c}\text { ration between wind } \\
\text { power and PV } \\
\text { power(\%) 50/50 }\end{array}$ & $\begin{array}{c}\text { ration between wind } \\
\text { power and PV } \\
\text { power(\%) 70/30 }\end{array}$ & $\begin{array}{l}\text { ration between wind } \\
\text { power and PV } \\
\text { power(\%) } 100 / 0\end{array}$ \\
\hline \multirow{3}{*}{$\begin{array}{l}\text { Early } \\
\text { in } \\
\text { morning }\end{array}$} & Probability of upper limit(\%) & 0.01 & 0.01 & 0.01 & 0.02 & 0.02 \\
\hline & Probability of lower limit(\%) & 0.25 & 0.15 & 0.1 & 0.06 & 0.02 \\
\hline & Total over-limit probability(\%) & 0.26 & 0.16 & 0.11 & 0.07 & 0.04 \\
\hline \multirow{3}{*}{ Morning } & Probability of upper limit(\%) & 0.26 & 0.07 & 0.04 & 0.03 & 0.01 \\
\hline & Probability of lower limit(\%) & 0 & 0 & 0 & 0 & 0.2 \\
\hline & Total over-limit probability(\%) & 0.26 & 0.07 & 0.04 & 0.03 & 0.21 \\
\hline \multirow{3}{*}{ Noon } & Probability of upper limit(\%) & 70.99 & 39.34 & 22.87 & 4.67 & 0.11 \\
\hline & Probability of lower limit(\%) & 0 & 0 & 0 & 0 & 0 \\
\hline & Total over-limit probability(\%) & 70.99 & 39.34 & 22.87 & 4.67 & 0.11 \\
\hline \multirow{2}{*}{$\begin{array}{l}\text { After- } \\
\text { noon }\end{array}$} & Probability of lower limit(\%) & 0.07 & 0.06 & 0.05 & 0.09 & 0.16 \\
\hline & Total over-limit probability(\%) & 0.12 & 0.11 & 0.09 & 0.17 & 0.19 \\
\hline \multirow{3}{*}{ Evening } & Probability of upper limit(\%) & 0 & 0 & 0.01 & 0.02 & 0.03 \\
\hline & Probability of lower limit(\%) & 40.67 & 15.36 & 1.94 & 0.38 & 0.03 \\
\hline & Total over-limit probability(\%) & 40.67 & 15.36 & 1.95 & 0.40 & 0.06 \\
\hline \multirow{3}{*}{ Night } & Probability of upper limit(\%) & 0 & 0.02 & 0.01 & 0.01 & 0.03 \\
\hline & Probability of lower limit(\%) & 51.34 & 10.02 & 0.24 & 0.06 & 0 \\
\hline & Total over-limit probability(\%) & 51.34 & 10.03 & 0.25 & 0.06 & 0.03 \\
\hline \multirow{2}{*}{$\begin{array}{c}\text { Late } \\
\text { at night }\end{array}$} & Probability of upper limit(\%) & 0.01 & 0.02 & 0.03 & 9.17 & 38.66 \\
\hline & Probability of lower limit(\%) & 0.21 & 0.02 & 0 & 0 & 0 \\
\hline
\end{tabular}

4) The laws of voltage limit violation of distribution line could be obtained by using the proposed calculation method comprehensively considering time-sequence characteristic and randomness, and the potential risks of the distribution lines could be found.

\section{REFERENCES}

[1] B. Li, T. Q. Liu and X. Y. Li, "Impact of Distributed Generation on Power System Voltage Stability,” Power System Technology, Vol. 33, No. 3, 2009, pp. 84-88

[2] H. N. Niu, X. Q. Huang, Z. Cai and M. G. Yang, "Probabilistic Evaluation of Voltage Limit Violation of Power System with Wind Power Generators Integrated,” Power System Protection and Control, Vol. 40, No. 18, 2012, pp. 1-6

[3] F. Chen, W. Wang, L. J. Xu, et al., "Analyzing the Voltage Variation of Distribution Network Including Distributed Generation,” Proceedings of the CSU-EPSA, , Vol.
24, No. 4, 2012, pp. 145-149

[4] X. Y. Xu, Y. H. Huang, C. Liu, et al., "Influence of Distributed Photovoltaic Generation on Voltage in Distribution Network and Solution of Voltage Beyond Limits," Power System Technology, Vol. 34, No. 10, 2010, pp. 140-146

[5] S. Conti, S. Raiti, G. Tina, et al., "Study of the Impact of PV Generationon Voltage Profile in LV Distribution Networks," 2001 IEEE Porto Power Technology Conference.

[6] J. Xiao, T. Q. Liu and P. Su, "Time-Interval Based Reactive Power Optimization Algorithm Using Double-Population Particle Swarm Optimization,” Power System Technology, Vol. 38, No. 8, 2009, pp. 72-77

[7] H. S. Liang, L. Cheng and S. Liu, "Monte Carlo Simulation Based Reliability Evaluation of Distribution System Containing Microgrids,” Power System Technology, Vol. 10, 2011, pp. 76-81. 